PROCEEDINGS OF THE

AMERICAN MATHEMATICAL SOCIETY

Volume 135, Number 9, September 2007, Pages 2803-2809

S $0002-9939(07) 08828-4$

Article electronically published on May 4, 2007

\title{
ORDER-WEAKLY COMPACT OPERATORS FROM VECTOR-VALUED FUNCTION SPACES TO BANACH SPACES
}

\author{
MARIAN NOWAK
}

(Communicated by Jonathan M. Borwein)

\begin{abstract}
Let $E$ be an ideal of $L^{0}$ over a $\sigma$-finite measure space $(\Omega, \Sigma, \mu)$, and let $E^{\sim}$ stand for the order dual of $E$. For a real Banach space $\left(X,\|\cdot\|_{X}\right)$ let $E(X)$ be a subspace of the space $L^{0}(X)$ of $\mu$-equivalence classes of strongly $\Sigma$-measurable functions $f: \Omega \longrightarrow X$ and consisting of all those $f \in L^{0}(X)$ for which the scalar function $\|f(\cdot)\|_{X}$ belongs to $E$. For a real Banach space $\left(Y,\|\cdot\|_{Y}\right)$ a linear operator $T: E(X) \longrightarrow Y$ is said to be order-weakly compact whenever for each $u \in E^{+}$the set $T(\{f \in E(X)$ : $\left.\left.\|f(\cdot)\|_{X} \leq u\right\}\right)$ is relatively weakly compact in $Y$. In this paper we examine order-weakly compact operators $T: E(X) \longrightarrow Y$. We give a characterization of an order-weakly compact operator $T$ in terms of the continuity of the conjugate operator of $T$ with respect to some weak topologies. It is shown that if $\left(E,\|\cdot\|_{E}\right)$ is an order continuous Banach function space, $X$ is a Banach space containing no isomorphic copy of $l^{1}$ and $Y$ is a weakly sequentially complete Banach space, then every continuous linear operator $T: E(X) \longrightarrow Y$ is order-weakly compact. Moreover, it is proved that if $\left(E,\|\cdot\|_{E}\right)$ is a Banach function space, then for every Banach space $Y$ any continuous linear operator $T: E(X) \longrightarrow Y$ is order-weakly compact iff the norm $\|\cdot\|_{E}$ is order continuous and $X$ is reflexive. In particular, for every Banach space $Y$ any continuous linear operator $T: L^{1}(X) \longrightarrow Y$ is order-weakly compact iff $X$ is reflexive.
\end{abstract}

\section{INTRODUCTION AND PRELIMINARIES}

P. G. Dodds D] considered order-weakly compact operators from a vector lattice $E$ to a Banach space $Y$. Recall that a linear operator $T: E \longrightarrow Y$ is called orderweakly compact if the set $T([-u, u])$ is relatively-weakly compact in $Y$ for every $u \in E^{+}$. Some further properties of these operators can be found in $\mathrm{M}$, Section 3.4]. M. Duhoux [Du extended Dodd's results to the setting $Y$ being a locally convex space. Z. Ercan $[\mathrm{E}$ examined some properties of order-weakly compact operators from a vector lattice $E$ to a topological vector space $Y$.

In this paper, we consider order-weakly compact operators from a vector-valued function space $E(X)$ to a Banach space $Y$.

Received by the editors December 18, 2003 and, in revised form, May 18, 2006.

2000 Mathematics Subject Classification. Primary 47B38, 47B07, 46E40, 46A20.

Key words and phrases. Vector-valued function spaces, Köthe-Bochner spaces, order-bounded operators, order-weakly compact operators, order intervals.

(C)2007 American Mathematical Society 
For terminology concerning Riesz spaces and function spaces we refer to AB1, AB2 and [KA]. Given a topological vector space $(L, \tau)$, by $(L, \tau)^{*}$ we will denote its topological dual. We denote by $\sigma(L, K), \beta(L, K)$ and $\tau(L, K)$ the weak topology, the strong topology and the Mackey topology, respectively, for a dual system $\langle L, K\rangle$. By $\mathbb{N}$ and $\mathbb{R}$ we will denote the sets of all natural and real numbers, respectively.

Throughout the paper we assume that $(\Omega, \Sigma, \mu)$ is a complete $\sigma$-finite measure space and $L^{0}$ denotes the corresponding space of $\mu$-equivalence classes of all $\Sigma$ measurable real-valued functions. Let $E$ be an ideal of $L^{0}$ with $\operatorname{supp} E=\Omega$ and let $E^{\sim}$ stand for the order dual of $E$.

Let $\left(X,\|\cdot\|_{X}\right)$ be a real Banach space, and let $S_{X}$ stand for the unit sphere of $X$. By $L^{0}(X)$ we denote the set of $\mu$-equivalence classes of all strongly $\Sigma$-measurable functions $f: \Omega \longrightarrow X$. For $f \in L^{0}(X)$ let us set $\widetilde{f}(\omega):=\|f(\omega)\|_{X}$ for $\omega \in \Omega$. Let

$$
E(X)=\left\{f \in L^{0}(X): \widetilde{f} \in E\right\} .
$$

For each $u \in E^{+}$the set $D_{u}=\{f \in E(X): \tilde{f} \leq u\}$ will be called an order interval in $E(X)$.

Following $[\mathrm{D}]$ we are now ready to define two classes of linear operators.

Definition 1.1. Let $E$ be an ideal of $L^{0}$, and let $\left(X,\|\cdot\|_{X}\right)$ and $\left(Y,\|\cdot\|_{Y}\right)$ be real Banach spaces. A linear operator $T: E(X) \longrightarrow Y$ is said to be order-weakly compact (resp. order-bounded) whenever for each $u \in E^{+}$the set $T\left(D_{u}\right)$ is relatively-weakly compact (resp. norm-bounded) in $Y$.

Clearly each order-weakly compact operator $T: E(X) \longrightarrow Y$ is order-bounded. Order-bounded operators $T: E(X) \longrightarrow Y$ have been considered in [N5].

Now we recall some terminology and results concerning the duality theory of the function spaces $E(X)$ as set out in [B1], [BL], [N1], [N2].

For a linear functonal $F$ on $E(X)$ let us put

$$
|F|(f)=\sup \{|F(h)|: h \in E(X), \widetilde{h} \leq \widetilde{f}\} \quad \text { for } \quad f \in E(X) .
$$

The set

$$
E(X)^{\sim}=\left\{F \in E(X)^{\#}:|F|(f)<\infty \quad \text { for all } \quad f \in E(X)\right\}
$$

will be called the order dual of $E(X)$ (here $E(X)^{\#}$ denotes the algebraic dual of $E(X))$.

For $F_{1}, F_{2} \in E(X)^{\sim}$ we will write $\left|F_{1}\right| \leq\left|F_{2}\right|$ whenever $\left|F_{1}\right|(f) \leq\left|F_{2}\right|(f)$ for all $f \in E(X)$. A subset $A$ of $E(X)^{\sim}$ is said to be solid whenever $\left|F_{1}\right| \leq\left|F_{2}\right|$ with $F_{1} \in E(X)^{\sim}$ and $F_{2} \in A$ imply $F_{1} \in A$.

In particular, for a Banach function space $\left(E,\|\cdot\|_{E}\right)$ the space $E(X)$ provided with the norm $\|f\|_{E(X)}:=\|\widetilde{f}\|_{E}$ is a Banach space, and it is usually called a KötheBochner space. It is well known that $\left(E(X),\|\cdot\|_{E(X)}\right)^{*}=E(X)^{\sim}$ (see [BL, §3, Lemma 12]).

For each $f \in E(X)$ let

$$
\rho_{f}(F)=|F|(f) \text { for } \quad F \in E(X)^{\sim} .
$$

We define the absolute weak topology $|\sigma|\left(E(X)^{\sim}, E(X)\right)$ on $E(X)^{\sim}$ as a locally convex topology generated by the family $\left\{\rho_{f}: f \in E(X)\right\}$ of seminorms. Clearly, $|\sigma|\left(E(X)^{\sim}, E(X)\right)$ is the topology of uniform convergence on the family of all order intervals $D_{u}$, where $u \in E^{+}$. 
Now let $\mathcal{B}_{0}$ be the family of all absolutely convex subsets of $E(X)$ that absorb every order interval in $E(X)$. Then $\mathcal{B}_{0}$ is a local base at zero for a locally convex topology $\tau_{0}$ on $E(X)$ (called an order-bounded topology), which is the finest locally convex topology on $E(X)$ for which every order interval is a bounded set (see $\mathrm{Na}$, N5).

The following characterization of $\tau_{0}$ and order-bounded operators $T: E(X) \rightarrow Y$ will be needed (see [N5, Corollary 2.2] and Theorem 2.3).

Theorem 1.2. The order-bounded topology $\tau_{0}$ on $E(X)$ coincides with the Mackey topology $\tau\left(E(X), E(X)^{\sim}\right)$, i.e., $\tau_{0}=\tau\left(E(X), E(X)^{\sim}\right)$. For a linear operator $T: E(X) \longrightarrow Y$ the following statements are equivalent:

(i) $T$ is order-bounded;

(ii) $T$ is $\left(\tau\left(E(X), E(X)^{\sim}\right),\|\cdot\|_{Y}\right)$-continuous;

(iii) $T$ is $\left(\sigma\left(E(X), E(X)^{\sim}\right), \sigma\left(Y, Y^{*}\right)\right)$-continuous.

Moreover, for a Banach function space $\left(E,\|\cdot\|_{E}\right)$, the statements (i)-(iii) are equivalent to the following:

(iv) $T$ is $\left(\|\cdot\|_{E(X)},\|\cdot\|_{Y}\right)$-continuous.

\section{Characterization OF ORDER-WeAKly COMPACT OPERATORS}

Let $\tau$ be a linear topology on $E(X)$. Recall that a linear operator $T: E(X)$ $\longrightarrow Y$ is $\tau$-weakly compact whenever there exists a neighbourhood $U$ of 0 for $\tau$ such that the set $T(U)$ is relatively-weakly compact in $Y$.

In view of the definition of the order-bounded topology $\tau_{0}$ on $E(X)$ we can easily observe that every $\tau_{0}$-weakly compact operator $T: E(X) \longrightarrow Y$ is orderweakly compact. Hence, if $\left(E,\|\cdot\|_{E}\right)$ is a Banach function space, then $\tau_{0}$ coincides with the $\|\cdot\|_{E(X)}$-topology, so every weakly compact operator $T: E(X) \longrightarrow Y$ is order-weakly compact. In particular, a linear operator $T: L^{\infty}(X) \longrightarrow Y$ is order-weakly compact if and only if $T$ is weakly compact (because the unit ball in $L^{\infty}(X)$ coincides with the order interval $D_{\mathbb{1}_{\Omega}}$ ).

In this section we characterize order-weakly operators $T: E(X) \longrightarrow Y$ in terms of the continuity of the conjugate operator $T^{\sim}$ with respect to appropriate weak topologies. We start by recalling some concepts and results of the duality theory of the spaces $E(X)$ (see [B1], [BL, [N1], [N2]).

For a linear functional $V$ on $E(X)^{\sim}$ let us put:

$$
|V|(F)=\sup \left\{|V(G)|: G \in E(X)^{\sim},|G| \leq|F|\right\} \quad \text { for } \quad F \in E(X)^{\sim} .
$$

The set

$$
\left(E(X)^{\sim}\right)^{\sim}=\left\{V \in\left(E(X)^{\sim}\right)^{\#}:|V|(F)<\infty \text { for all } F \in E(X)^{\sim}\right\}
$$

will be called the order dual of $E(X)^{\sim}$ (here $\left(E(X)^{\sim}\right)^{\#}$ denotes the algebraic dual of $\left.E(X)^{\sim}\right)$.

For $V_{1}, V_{2} \in\left(E(X)^{\sim}\right)^{\sim}$ we will write $\left|V_{1}\right| \leq\left|V_{2}\right|$ whenever $\left|V_{1}\right|(F) \leq\left|V_{2}\right|(F)$ for all $F \in E(X)^{\sim}$. A subset $K$ of $\left(E(X)^{\sim}\right)^{\sim}$ is said to be solid whenever $\left|V_{1}\right| \leq\left|V_{2}\right|$ with $V_{1} \in\left(E(X)^{\sim}\right)^{\sim}, \quad V_{2} \in K$ imply $V_{1} \in K$. A linear subspace $L$ of $\left(E(X)^{\sim}\right)^{\sim}$ is called an ideal of $\left(E(X)^{\sim}\right)^{\sim}$ if $L$ is a solid subset of $\left(E(X)^{\sim}\right)^{\sim}$.

For each $f \in E(X)$ let us put

$$
\pi_{f}(F)=F(f) \text { for all } F \in E(X)^{\sim} .
$$


One can show (see [N2]) that for $f \in E(X)$,

$$
\left|\pi_{f}\right|(F)=|F|(f) \text { for } F \in E(X)^{\sim} \quad \text { and } \quad \pi_{f} \in\left(E(X)^{\sim}\right)^{\sim} .
$$

Thus we have a natural embedding $\pi: E(X) \ni f \longmapsto \pi_{f} \in\left(E(X)^{\sim}\right)^{\sim}$. Denote by $E(X)_{0}$ the ideal of $\left(E(X)^{\sim}\right)^{\sim}$ generated by the set $\pi(E(X))$ (i.e., $E(X)_{0}$ is the smallest ideal of $\left(E(X)^{\sim}\right)^{\sim}$ containing $\left.\pi(E(X))\right)$.

Theorem 2.1 (see [N2, Theorem 3.2]). We have

$$
\begin{aligned}
&\left(E(X)^{\sim},|\sigma|\left(E(X)^{\sim}, E(X)\right)\right)^{*}=E(X)_{0} \\
& \quad=\left\{V \in\left(E(X)^{\sim}\right)^{\sim}:|V| \leq\left|\pi_{f}\right| \quad \text { for some } f \in E(X)\right\} .
\end{aligned}
$$

For $u \in E^{+}$and $f \in E(X)$ let:

$$
\begin{gathered}
C_{u}:=\pi\left(D_{u}\right)=\left\{\pi_{h}: h \in E(X), \widetilde{h} \leq u\right\} \quad(=\text { an interval in } \pi(E(X))), \\
I_{f}:=\left\{V \in E(X)_{0}:|V| \leq\left|\pi_{f}\right|\right\} \quad\left(=\text { an interval in } E(X)_{0}\right) .
\end{gathered}
$$

The following properties of $C_{u}$ and $I_{f}$ will be needed.

Theorem 2.2 ([르, Theorem 2.4]). Let $f \in E(X)$. Then

(i) $I_{f}$ is $\sigma\left(E(X)_{0}, E(X)^{\sim}\right)$-compact in $E(X)_{0}$;

(ii) $C_{\tilde{f}}$ is $\sigma\left(E(X)_{0}, E(X)^{\sim}\right)$-dense in $I_{f}$.

Assume now that a linear operator $T: E(X) \longrightarrow Y$ is order-bounded. Then in view of Theorem 1.1, $T$ is $\left.\left(\sigma\left(E(X), E(X)^{\sim}\right), \sigma\left(Y, Y^{*}\right)\right)\right)$-continuous. Hence, we can consider the linear mapping

$$
T^{\sim}: Y^{*} \longrightarrow E(X)^{\sim}
$$

defined by

$$
T^{\sim}\left(y^{*}\right)(f)=y^{*}(T(f)) \quad \text { for } \quad y^{*} \in Y \quad \text { and all } \quad f \in E(X) .
$$

Then $T^{\sim}$ is $\left(\beta\left(Y^{*}, Y\right), \beta\left(E(X)^{\sim}, E(X)\right)\right)$-continuous; hence $T^{\sim}$ is also $\left(\beta\left(Y^{*}, Y\right), \quad|\sigma|\left(E(X)^{\sim}, E(X)\right)\right)$-continuous ( because $|\sigma|\left(E(X)^{\sim}, E(X)\right) \subset$ $\beta\left(E(X)^{\sim}, E(X)\right)$; see $([\mathbb{N 2}, \S 4])$. Since $\left(E(X)^{\sim},|\sigma|\left(E(X)^{\sim}, E(X)\right)\right)^{*}=E(X)_{0}$ (see Theorem 2.1) and $\left(Y^{*}, \beta\left(Y^{*}, Y\right)\right)^{*}=Y^{* *}$, we see that $T^{\sim}$ is also $\left(\sigma\left(Y^{*}, Y^{* *}\right), \sigma\left(E(X)^{\sim}, E(X)_{0}\right)\right)$-continuous. Finally, we consider the linear mapping

defined by

$$
T^{\sim \sim}: E(X)_{0} \longrightarrow Y^{* *}
$$

$$
T^{\sim \sim}(V)\left(y^{*}\right)=V\left(T^{\sim}\left(y^{*}\right)\right) \quad \text { for } \quad V \in E(X)_{0} \quad \text { and all } \quad y^{*} \in Y^{*},
$$

which is $\left(\sigma\left(E(X)_{0}, E(X)^{\sim}\right), \sigma\left(Y^{* *}, Y^{*}\right)\right)$-continuous.

Let $i: Y \ni y \longmapsto i_{y} \in Y^{* *}$ stand for the canonical isometry, i.e.,

$$
i_{y}\left(y^{*}\right)=y^{*}(y) \text { for } y^{*} \in Y^{*} .
$$

It is seen that $T^{\sim \sim} \circ \pi=i \circ T$, where $\pi: E(X) \longrightarrow\left(E(X)^{\sim}\right)^{\sim}$ is a natural embedding.

Now we are ready to state a "vector-valued version" of a characterization of order-weakly compact operators $T: E \longrightarrow Y$ (see [D, Theorem 4.2]).

Theorem 2.3. For an order-bounded operator $T: E(X) \longrightarrow Y$ the following statements are equivalent:

(i) $T$ is order-weakly compact; 
(ii) $T^{\sim \sim}\left(E(X)_{0}\right) \subset i(Y)$;

(iii) $T^{\sim}:\left(Y^{*}, \sigma\left(Y^{*}, Y\right)\right) \longrightarrow\left(E(X)^{\sim}, \sigma\left(E(X)^{\sim}, E(X)_{0}\right)\right)$ is continuous.

Proof. (i) $\Longrightarrow$ (ii) Assume that $T$ is order-weakly compact and let $f \in E(X)$. Since the set $C_{\widetilde{f}}$ is $\sigma\left(E(X)_{0}, E(X)^{\sim}\right)$-dense in $I_{f}$ (see Theorem 2.2), by the $\left(\sigma\left(E(X)_{0}, E(X)^{\sim}\right), \sigma\left(Y^{* *}, Y^{*}\right)\right)$-continuity of $T^{\sim \sim}$ we obtain

$$
T^{\sim \sim}\left(I_{f}\right)=T^{\sim \sim}\left(c l_{\sigma\left(E(X)_{o}, E(X) \sim\right)} C_{\widetilde{f}}\right) \subset c l_{\sigma\left(Y^{* *}, Y^{*}\right)} T^{\sim \sim}\left(C_{\widetilde{f}}\right) .
$$

But for $h \in E(X)$ and $y^{*} \in Y^{*}$ we have

$$
T^{\sim \sim}\left(\pi_{h}\right)\left(y^{*}\right)=\pi_{h}\left(T^{\sim}\left(y^{*}\right)\right)=\pi_{h}\left(y^{*} \circ T\right)=y^{*}(T(h))=i_{T(h)}\left(y^{*}\right) .
$$

Thus putting $A_{f}=T(\{h \in E(X): \widetilde{h} \leq \tilde{f}\})$ we get

$$
T^{\sim \sim}\left(C_{\widetilde{f}}\right)=\left\{i_{T(h)}: h \in E(X), \widetilde{h} \leq \widetilde{f}\right\}=i(T(\{h \in E(X): \widetilde{h} \leq \widetilde{f}\}))=i\left(A_{f}\right) .
$$

Since $T$ is order-weakly compact, the set $A_{f}$ is relatively $\sigma\left(Y, Y^{*}\right)$-compact in $Y$, so the space $\left(c l_{\sigma\left(Y, Y^{*}\right)} A_{f},\left.\sigma\left(Y, Y^{*}\right)\right|_{c l_{\sigma\left(Y, Y^{*}\right)} A_{f}}\right)$ is compact. Hence in view of the $\left(\sigma\left(Y, Y^{*}\right), \sigma\left(Y^{* *}, Y^{*}\right)\right)$-continuity of $i$ we obtain that the set $i\left(c l_{\sigma\left(Y, Y^{*}\right)} A_{f}\right)$ is $\sigma\left(Y^{* *}, Y^{*}\right)$-closed in $Y^{* *}$. It follows that

$$
c l_{\sigma\left(Y^{* *}, Y^{*}\right)} i\left(A_{f}\right) \subset i\left(c l_{\sigma\left(Y, Y^{*}\right)} A_{f}\right) \subset i(Y),
$$

so finally $T^{\sim \sim}\left(I_{f}\right) \subset i(Y)$. Hence, since $E(X)_{0}=\bigcup\left\{I_{f}: f \in E(X)\right\}$, we conclude that $T^{\sim \sim}\left(E(X)_{0}\right) \subset i(Y)$.

(ii) $\Longrightarrow$ (i) Assume that $T^{\sim \sim}\left(E(X)_{0}\right) \subset i(Y)$ and let $u \in E^{+}$. Let us set $f=u \otimes x_{0}$, where $x_{0} \in S_{X}$ and $\left(u \otimes x_{0}\right)(\omega)=u(\omega) x_{0}$ for $\omega \in \Omega$. Since the mapping $T^{\sim \sim}: E(X)_{0} \longrightarrow Y^{* *}$ is $\left(\sigma\left(E(X)_{0}, E(X)^{\sim}\right), \sigma\left(Y^{* *}, Y^{*}\right)\right)$-continuous and $I_{f}$ is a $\sigma\left(E(X)_{0}, E(X)^{\sim}\right)$-compact subset of $E(X)_{0}$, we obtain that the set $T^{\sim \sim}\left(I_{f}\right)$ is a $\sigma\left(i(Y), Y^{*}\right)$-compact subset of $i(Y)$. Since $T^{\sim \sim} \circ \pi=i \circ T$ and $\pi\left(D_{u}\right)=C_{\widetilde{f}}$, we get $i\left(T\left(D_{u}\right)\right)=T^{\sim \sim}\left(\pi\left(D_{u}\right)\right)=T^{\sim \sim}\left(C_{\widetilde{f}}\right) \subset T^{\sim \sim}\left(I_{f}\right)$. Hence $i\left(T\left(D_{u}\right)\right)$ is a relatively compact subset of $\left(i(Y), \sigma\left(i(Y), Y^{*}\right)\right)$, so $T\left(D_{u}\right)$ is relatively-weakly compact in $Y$.

(ii) $\Longleftrightarrow$ (iii) See [W, Lemma 11.1.1].

\section{ORDER-WEAKLY COMPACT OPERATORS From KÖTHE-BOCHNER SPACES TO BANACH SPACES}

In this section we assume that $\left(E,\|\cdot\|_{E}\right)$ is a Banach function space. Then $E(X)^{\sim}=E(X)^{*}\left(=\left(E(X),\|\cdot\|_{E(X)}\right)^{*}\right)$. In view of Theorem 1.1 for a Banach space $Y$ a linear operator $T: E(X) \longrightarrow Y$ is order-bounded if and only if $T$ is continuous (i.e., $\left(\|\cdot\|_{E(X)},\|\cdot\|_{Y}\right)$-continuous).

Recall that a subset $A$ of the Banach space is said to be conditionally-weakly compact whenever each sequence in $A$ contains a weakly Cauchy subsequence.

Making use of [N3, Corollary 2.8] we get:

Theorem 3.1. Let $\left(E,\|\cdot\|_{E}\right)$ be an order continuous Banach function space and $X$ a Banach space containing no isomorphic copy of $l^{1}$. Then for each $u \in E^{+}$ the order interval $D_{u}$ is conditionally-weakly compact in $E(X)$.

As an application of Theorem 3.1 we get: 
Theorem 3.2. Let $\left(E,\|\cdot\|_{E}\right)$ be an order continuous Banach function space, $X$ a Banach space containing no isomorphic copy of $l^{1}$ and $Y$ a weakly sequentially complete Banach space. Then every continuous linear operator $T: E(X) \longrightarrow Y$ is order-weakly compact.

Proof. Let $u \in E^{+}$. Then by Theorem 3.1, $D_{u}$ is a conditionally-weakly compact set in $E(X)$. It follows that the set $T\left(D_{u}\right)$ is conditionally-weakly compact in $Y$. Since $Y$ is supposed to be weakly sequentially complete, we conclude that $T\left(D_{u}\right)$ is relatively-weakly sequentially compact in $Y$. Hence $T\left(D_{u}\right)$ is relatively-weakly compact in $Y$.

In particular, we have:

Corollary 3.3. Let $X$ be a Banach space containing no isomorphic copy of $l^{1}$ and let $Y$ be a weakly sequentially complete Banach space. Then every continuous linear operator $T: L^{p}(X) \longrightarrow Y(1 \leq p<\infty)$ is order-weakly compact.

It is well known that a Lebesgue-Bochner space $L^{q}(Y) \quad(1 \leq q<\infty)$ is weakly sequentially complete whenever a Banach space $Y$ is weakly sequentially complete (see [T, Theorem 11]). Hence, as a consequence of Corollary 3.3 we get:

Corollary 3.4. Let $X$ be a Banach space containing no isomorphic copy of $l^{1}$ and let $Y$ be a weakly sequentially complete Banach space. Then every continuous linear operator $T: L^{p}(X) \longrightarrow L^{q}(Y) \quad(1 \leq p<\infty, 1 \leq q<\infty)$ is order-weakly compact.

Now, we present necessary and sufficient conditions for the order-weak compactness of any continuous operator $T: E(X) \longrightarrow Y$ with any Banach space $Y$. For this purpose we shall need the following characterization of weak compactness of order intervals in $E(X)$.

Theorem 3.5 (see [BL, §4, Corollary 1], B2, Proposition 2], [N4, Theorem 2.4]). Let $\left(E,\|\cdot\|_{E}\right)$ be a Banach function space and $X$ a Banach space. Then the following statements are equivalent:

(i) the norm $\|\cdot\|_{E}$ is order continuous and $X$ is reflexive;

(ii) for each $u \in E^{+}$the order interval $D_{u}$ is weakly compact.

Theorem 3.6. Let $\left(E,\|\cdot\|_{E}\right)$ be a Banach function space and $X$ a Banach space. Then the following statements are equivalent:

(i) the norm $\|\cdot\|_{E}$ is order continuous and $X$ is reflexive;

(ii) for every Banach space $Y$, any continuous linear operator $T: E(X) \longrightarrow Y$ is order-weakly compact.

Proof. (i) $\Longrightarrow$ (ii) This follows from Theorem 3.5.

(ii) $\Longrightarrow$ (i) Assume that (ii) holds and let $\left(Y,\|\cdot\|_{Y}\right)=\left(E(X),\|\cdot\|_{E(X)}\right)$. Then the identity operator id $: E(X) \longrightarrow E(X)$ is order-weakly compact. It follows that for each $u \in E^{+}$the set $D_{u}$ is weakly compact in $E(X)$, so by Theorem 3.5 the norm $\|\cdot\|_{E}$ is order continuous and $X$ is reflexive.

In particular, as an application of Theorem 3.6 we get:

Corollary 3.7. For a Banach space $X$ the following statements are equivalent:

(i) $X$ is reflexive;

(ii) for every Banach space $Y$, any continuous linear operator $T: L^{p}(X) \longrightarrow Y$ $(1 \leq p<\infty)$ is order-weakly compact. 


\section{REFERENCES}

[AB1] C. D. Aliprantis, O. Burkinshaw, Locally solid Riesz spaces, Academic Press, New York, San Francisco, London, 1978. MR0493242 (58:12271)

[AB2] C. D. Aliprantis, O. Burkinshaw, Positive operators, Academic Press, Orlando, San Diego, New York, London, Tokyo, 1985. MR0809372 (87h:47086)

[B1] A. V. Bukhvalov, On an analytic representation of operators with abstract norm, Izv. Vyss. Vceb. Zaved., 11 (1975), 21-32 (in Russian). MR0470746 (57:10492)

[B2] A. V. Bukhvalov, Factorization of linear operators in Banach lattices and in spaces of vector-valued functions, in Qualitative and approximate methods for investigating operator equations, 168, 34-46, Yaroslav Gos. Univ., Yaroslav, 1982 (in Russian). MR0857126 (87m:47049)

[BL] A. V. Bukhvalov, G. Ya. Lozanowskii, On sets closed in measure in spaces of measurable functions, Trans. Moscow Math. Soc., 2 (1978), 127-148.

[D] P. G. Dodds, o-weakly compact mappings of Riesz spaces, Trans. Amer. Math. Soc., 214 (1975), 389-402. MR0385629 (52:6489)

[Du] M. Duhoux, o-weakly compact mappings from a Riesz space to a locally convex space, Bull. Math. Soc. Sci. Math. R. S. Roumanie, 22 (70) no. 4 (1978), 371-378. MR0522533 (80a:47057)

[E] Z. Ercan, Interval-bounded operators and order-weakly compact operators of Riesz spaces, Demonstr. Math., 31, no. 4 (1998), 805-812. MR.1677834 (2000g:47046)

[KA] L. V. Kantorovitch, A. V. Akilov, Functional Analysis, Nauka, Moscow, 1984 (3 ${ }^{\text {rd }}$ ed., in Russian). MR0788496 (86m:46001)

[M] P. Meyer-Nieberg, Banach Lattices, Springer Verlag, Berlin, Heidelberg, 1991. MR1128093 (93f:46025)

[Na] I. Namioka, Partially ordered linear topological spaces, Mem. Amer. Math. Soc., no. 24 (1957). MR0094681 (20:1193)

[N1] M. Nowak, Duality theory of vector-valued function spaces I, Comment. Math., Prace Mat., 37 (1997), 195-215. MR1608189 (99h:46061)

[N2] M. Nowak, Duality theory of vector-valued function spaces II, Comment. Math., Prace Mat., 37 (1997), 217-230. MR1608185 (99h:46062)

[N3] M. Nowak, Weak sequential compactness and completeness in Köthe-Bochner spaces, Bull. Polish Acad. Sci. Math., 47, no. 3 (1999), 209-220. MR.1711823 (2000h:46050)

[N4] M. Nowak, On some topological properties of vector-valued function spaces, Rocky Mountain J. Math. (to appear).

[N5] M. Nowak, Order bounded operators from vector-valued function spaces to Banach spaces, Proc. Conf. Function Spaces VII, Poznań 2003, Banach Center Publ., 68 (2005), 109-114. MR2179004 (2006e:47067)

[T] M. Talagrand, Weak Cauchy sequences in $L^{1}(E)$, Amer. J. Math., 106 (1984), 703-724. MR0745148(85j:46062)

[W] A. Wilansky, Modern methods in topological vector spaces, McGraw-Hill Inc., 1978. MR0518316 (81d:46001)

Faculty of Mathematics, Computer Science and Econometrics, University of Zielona Góra, ul. Szafrana 4A, 65-001 Zielona Góra, Poland

E-mail address: M.Nowak@wmie.uz.zgora.pl 